Journal of

Women's Health and Gynecology

\title{
Diagnosis of Bacterial Vaginosis Using a Novel Molecular Real-Time PCR Test
}

Karin Breding ${ }^{1}$, MDIris Vikström ${ }^{1}$, Assistant research nurse ${ }^{2}$, Anders Selbing², MD, PhD, Malin Farnebäck ${ }^{3}$, PhD, Alexandra Hermelin ${ }^{3}$ and Per-Göran Larsson ${ }^{1, *}, \mathrm{MD}, \mathrm{PhD}$

${ }^{1}$ Department of Obstetrics and Gynaecology, Skaraborgs Hospital,Skövde, SE-541 42 Skövde, Sweden

${ }^{2}$ Department of clinical and experimental medicine, IKE, University Hospital Linköping, SE- 58183 Linköping, Sweden

${ }^{3}$ Dynamic Code AB, Linköping, SE-582 16, Sweden

*Corresponding author: Per-Göran Larsson, Department of Obstetrics and Gynaecology, Skaraborgs Hospital, SE-541 42Skövde, Tel: +46500431445; Email:pg.larsson@vgregion.se

Received Date: January 02, 2020 Accepted Date: January 23, 2020 Published Date: January 25, 2020

Citation: Karin Breding (2020) Diagnosis of Bacterial Vaginosis Using a Novel Molecular Real-Time PCR Test. J Womens Health Gyn 7: 1-7.

\begin{abstract}
Background: Bacterial vaginosis is commonly diagnosed using either Amsel's or Nugent's criteria. These diagnostic tests are somewhat subjective and there is a need for more objective and reliable tests for the diagnosis of bacterial vaginosis.

Methods: A longitudinal study was conducted at a hospital in Sweden during 2012-2013 involving 300 pregnant women seeking legal abortion was conducted at a hospital in Sweden. Bacterial vaginosis was determined to be absent or present on the basis of a modified Hay/Ison criteria assessment and compared with a molecular test analyzing six different bacteria associated with bacterial vaginosis (A. vaginae, BVAB2, G. vaginalis, Leptotrichia/Sneathia spp., Megasphaera spp., and Mobiluncus spp.) in relation to Lactobacillus spp. using real-time PCR. The Cohen kappa coefficient test was used to determine the measure of agreement between the two diagnostic tests.

Results: This study showed that there is an excellent agreement between the compared methods, with a kappa coefficient value of 0.87 (0.76-0.99). As compared to the modified Hay/Ison critera, the molecular test achieved a sensitivity of $91 \%$, specificity of $97 \%$, positive predictive value of $91 \%$ and negative predictive value of $97 \%$.

Conclusions: The molecular test, using six different pathogens in an algorithm comparing their presence with that of lactobacilli, can accurately diagnose bacterial vaginosisin a clinical setting. The molecular test performed comparably to wet mount microscopy of vaginal swab samples. If the molecular test will be equally as effective when used as a "test of cure" needs to be investigated.
\end{abstract}

Trial registration: Clinical trial registration \# NCT04067557, retrospectively registered.

Keywords: Vaginosis; bacterial; Diagnosis; Molecular Diagnostic Techniques; Polymerase Chain Reaction.

Abbreviations: BV: Bacterial vaginosisgestational hypertension; PE: preeclampsia; AII: angiotensin II; ASA: acetyl-salicylic acid; IVF: in-vitro fecundation

(C2020 The Authors. Published by the JScholar under the terms of the Creative Commons Attribution License http://creativecommons.org/licenses/ by $/ 3.0 /$, which permits unrestricted use, provided the original author and source are credited. 
Key message: Diagnosis of bacterial vaginosis using a molecular test based on self-sampled vaginal swab is comparable to diagnosis by Hay/Ison criteria.

\section{Background}

Bacterial vaginosis (BV) is a disturbance in the bacterial flora of the vagina. It is prevalent in fertile women, occurring only rarely in post-menopausal women. BV is the most common cause of abnormal vaginal discharge and diffuse vaginal problems. The etiology is still unknown but is most certainly multifactorial [1]. BV decreases the presence of lactobacilli, consequently raising the $\mathrm{pH}$ in the vagina, often to a $\mathrm{pH}$ level between 5.5-6.0, beyond the normal healthy vaginal $\mathrm{pH}$ level range of 3.8-4.5.

There is no simple and easy test to diagnose BV as there is no single bacterial strain attributable to BV infection. The most commonly used method for diagnosis of BV are the Amsel criteria [2], where it is necessary to demonstrate three out of four of the following clinical criteria in order to accurately diagnose BV, including: typical homogeneous vaginal discharge, elevated $\mathrm{pH}$ level of the vaginal secretion (above $\mathrm{pH} 4.5$ ), positive amine test (whiff test) and the presence of clue cells. The most notable disadvantages of Amsel's criteria is the requirement of a clinical examination of the woman and the unavoidable degree of subjectivity in the assessment. The results are, thus, not easy to replicate. Therefore, other diagnostic tests have been introduced, including another commonly used method, the Nugent score [3], in which a Gram stain is performed on the vaginal sample and is subsequently investigated under a microscope at a magnification of 1000x. The final score for the Nugent scoring system for Gram stained vaginal smears is acquired by adding the Lactobacillus content score to the Gardnerella content score. Lactobacilli are evaluated and scored $0,1,2,3$, or 4 based on the average number of lactobacilli observed across multiple fields, where by the presence of more than 30 lactobacilli gives a score of 0,5-30 lactobacilli gives a score of 1, 1-4 lactobacilli gives a score of 2 , an average score of less than 1 lactobacilli gives a score of 3 , and no lactobacilli observed per visual field gives a score of 4. Gardnerella-like bacteria are scored in a similar manner but in the reverse order, where the absence of Gardnerella-like bacteria gives a score of 0 and more than 30 Gardnerella-like bacteria gives a score of 4 . If curved rods - Mobiluncus - are present the score is increased by a score of 1-2 depending on the number of Mobiluncus observed. The scores are then added together to obtain a final score. Thus, a score of $0-3$ indicates normal lactobacilli flora, a score of 4-6 indicates intermediate levels of bacterial flora, and a score greater than 7 indicates a bacterial flora consistent with a BV diagnosis.

Nugent's method of BV diagnosis also suffers from some disadvantages. Firstly, the vaginal smears are scored by quantification of the different vaginal morphotypes, which requires an experienced laboratory technician and microscopist as well as considerable time and skill $[4,5]$. Secondly, the microscope area can differ by as much as $300 \%$ between different microscopes [6], eg 30 lactobacilli can be viewed in the field of view of a narrow-angle microscope compared to 90 in the field of view of a wide-angle microscope.

A simpler method was described by Hay et al. [7, 8]. The Hay/Ison criteria is based on the inspection of Gram stains, performed to estimate the ratio of the observed morphotypes rather than determine the number of bacteria present, where observations are divided into three grades: grade 1 (normal - many lactobacilli morphotypes-few gardnerella morphotypes), grade 2 (intermediate - equal numbers of lactobacilli and gardnerella morphotypes), and grade 3 (BV - few lactobacilli and many gardnerella morphotypes). As with Nugent's score this method is time-consuming and requires skilled personnel.

All of the currently used methods for the diagnosis of BV have their disadvantages. Thus, the need of faster, more cost-effective and objective methods still remains. Different molecular methods have been introduced in the diagnosis of BV [912]. Just recently Schwebke et al. published a large study comparing the molecular method BD MAX vaginal panel from Becton Dickinson to Amsel's criteria and Nugent's score [13]. The results are promising and show better diagnostic value than the aforementioned traditional clinical diagnostic tests.

Under routine procedure, in Sweden women scheduled for an abortion are screened for bacterial infections including Chlamydia trachomatis, Mycoplasma genitalium and Neisseria gonorrhoeae, together with a vaginal sample examined for the presence of BV [14].

For women admitted into hospital for an abortion there are two major strategies for reducing postoperative infections. Patients are either given antibiotic prophylaxis [14] or are screened and treated for specific for bacterial infections in order to reduce post-abortion infections [15]. Our clinic also screens for bacterial vaginos is prior to abortions. Thus, the objective of the study is to compare the use of a modified Hay/Ison criteria applied to air-dried vaginal and rehydrated wet smears with a 
new molecular test for diagnosis of BV in pregnant women.

\section{Methods}

\section{Participants}

Women were recruited to this study from a cohort comprised of pregnant women admitted into the out-patient clinic in the gynecological department of Skaraborgs Hospital in Skövde, Sweden, for a legal abortion procedure. The study was conducted over a period of four consecutive months over the course of 2012-2013, collecting vaginal samples from 300 women in total. A pelvic examination and an endovaginal ultrasound were performed and samples were taken for PCR analysis for Chlamydia trachomatis, Mycoplasma genitalium and Neisseria gonorrhoeae together with two vaginal samples for the diagnosis of BV.

\section{Microscopy}

The first of the set of two vaginal samples obtained from the woman for the purpose of BV diagnosis was used for microscope analysis by first transferring the vaginal sample onto a glass microscope slide. The slide was allowed to air-dry and, after adding saline, examined under 400x magnification using a Double Binocular Carl Zeiss Axiostar Plus phase contrast microscope (Carl Zeiss AG, Oberkochen, Germany) [16]. The slides were evaluated using a modified Hay/Ison criteria [17]. The observations were divided into five grades: grade 0 (vaginal smears free of bacteria), grade 1 (normal - many lactobacilli morphotypes-few Gardnerella morphotypes), grade 2 (intermediate - equal numbers of lactobacilli and Gardnerella morphotypes), grade 3 (BV - few lactobacilli and many Gardnerella morphotypes), and grade 4 (large concentrations of Gram-positive cocci, i.e. Streptococcus spp. or Staphylococcus spp. morphotypes). For comparison the microscope results were divided into BV positive (grade 3 ) and non-BV (grade 1 ). The samples with grade 0,2 and 4 are merged to the non-BV group (grade 1).

\section{Molecular test}

The second vaginal sample obtained from the women for the purpose of BV diagnosis was collected using FLOQSwabs ${ }^{\mathrm{Tm}}$ (Copan, Brescia, Italy) and analyzed by quantitative (q) PCR for A. vaginae, BVAB2, G. vaginalis, Lactobacillus spp., Leptotrichial Sneathia spp., Megasphaera spp., and Mobiluncus spp. The analysis was performed by Dynamic Code AB in Linköping, Sweden.

In short, DNA was extracted from the vaginal swabs using ZR-96 Quick-g DNA ${ }^{\mathrm{Tm}}$ (Zymo Research, Irvine, CA, USA).
The cellular material in the vaginal swab was lysed in $300 \mu \mathrm{l}$ Genomic Lysis Buffer. $50 \mu \mathrm{l}$ of the sample was diluted further in $150 \mu \mathrm{l}$ Genomic Lysis Buffer and disrupted in a TissueLyser (Qiagen, Hilden, Germany) for $2 \mathrm{~min}$ at $30 \mathrm{~Hz}$, and $100 \mu \mathrm{l}$ was subsequently applied on a Silicon- $\mathrm{A}^{\mathrm{Tw}}$ Plate and washed according to the manufacturer's instructions. DNA was eluted in $30 \mu$ Elution Buffer.

Primers and TaqMan MGB probes were designed to anneal to the rRNA gene of A. vaginae, BVAB2, G. vaginalis, Lactobacillus spp., Leptotrichia/Sneathia spp., Megasphaera spp., and Mobiluncus spp. TaqMan probes were labelled with either 6-FAM, VIC or NED to enable multiplex PCR reactions. Oligonucleotides were purchased from Thermo Fisher (Foster City, CA, USA). The analysis was made in three reactions: two multiplex reactions, one for $A$. vaginae, $G$. vaginalis and Leptotrichia/Sneathia spp. and the other for BVAB2, Megasphaera spp., and Mobiluncus spp; and one singleplex reaction for Lactobacillus spp., respectively. Each PCR reaction was performed in PerfeCTa ${ }^{\circledR}$ MultiPlex qPCR SuperMix (Quanta Biosciences, Inc, Gaithersburg, MD, USA) in a total volume of $15 \mu \mathrm{l}$ for each reaction. Template DNA volume used was 3 $\mu$ l. Analysis was performed on an ABI Prism 7300 Sequence Detection System (Life Technologies Corp, Carlsbad, CA, USA).

Before further interpretation of the results, the individual results from the analyses of each of the bacteria associated with BV (A. vaginae, G. vaginalis, Leptotrichia/Sneathia spp., BVAB2, Megasphaera spp., and Mobiluncus spp.) were "normalized" by subtracting the $C_{t}$ value for each species with the $\mathrm{C}_{\mathrm{t}}$ value from the Lactobacillus spp. analysis. Each individual result was then combined to produce a final result according to an algorithm developed by Dynamic Code AB.

\section{Statistical analyses}

To assess the reliability of the molecular testin comparison to the modified Ison/Hay diagnostic test a kappa value $(\kappa)$ and the associated $95 \%$ confidence intervals were calculated. Cohen's kappa value shows correlation between two methods and considers the possibility of coincidence. The calculations give a value between $<0,00-1,00$. Values of kappa were categorized based on the amount of agreement they suggest as follows: $\kappa>$ 0.75 represents excellent agreement, $0.40 \leq \kappa \leq 0.75$ represents fair to good agreement, and $\kappa<0.40$ represents poor agreement [18].The statistics were calculated using the Open Epi Software. 


\section{Ethics approval and consent to participate}

Ethical approval for the study was obtained from the regional ethics committee (EPN) for Gothenburg (EPN reference number 658-09 entitled "Molecular biological methods to verify STD-agents") on 21 January 2010. Participants consented to participate through providing verbal informed consent before their inclusion in the study.

Clinical trail registration \# NCT04067557, retrospectively registered.

\section{Results}

Vaginal swabs were collected from 300 women who had consented to participate in the study. Out of the twelve incomplete sets four lacked sufficient biological material in the wet smears, one contained too many red blood cells to be evaluated by wet smear, and seven lacked either the wet smear or PCR sample. This mean that the study was based on 288 samples that both had a wet smear and a PCR test.

In the wet smear analysis, the majority of the samples were scored as grade $1(n=199)$, six were grade 0 , three were grade 2 and none were grade 4 . These 208 vaginal samples (72\%) were merged to form the "non-BV" group. The remaining 80 vaginal samples $(28 \%)$ were scored as grade 3 , forming the "BV positive" group.

The molecular test was normal in 212women (74\%) and indicated BV in 76 women (26\%). Comparing the two methods gives a kappa coefficient value of 0.82 with a confidence interval of $0.71-0.94$, and the molecular test attained a sensitivity of 0.89 , a specificity of 0.94 , a positive predictive value of 0.85 and a negative predictive value of 0.96 (Table 1 ).

\begin{tabular}{|l|l|l|l|l|}
\hline & \multicolumn{3}{|c|}{ Molecular test } & \\
\hline & & BV & Normal & \\
\hline \multirow{2}{*}{$\begin{array}{l}\text { Modified Hay/ } \\
\text { Ison }\end{array}$} & BV & 68 & 12 & 80 \\
\cline { 2 - 6 } & Normal & 8 & 200 & 208 \\
\hline & & 76 & 212 & 288 \\
\hline
\end{tabular}

Table 1. The kappa coefficient value was calculated $\kappa=0.82$ (0.71-0.94) between a modified Hay/Ison criteria for the diagnosis of BV compared with a molecular test using six different pathogens and an algorithm using the presence of lactobacilli. This gives a sensitivity of $0.89,(0.81-0.95)$ a specificity of 0.94
(0.90-0.98), positive predictive value of $0.85(0.76-0.91)$ and negative predictive value of 0.96 (0.93-0.98).

Twenty samples gave discordant results, where twelve samples gave a positive wet smear result for BV but obtained a negative PCR test result and eight samples gave a negative wet smear result but obtained a positive PCR test result. The wet smears of these twenty sample sets were re-evaluated by microscope. This second evaluation was made simultaneously by two gynecologists, of which one is a highly experienced diagnostician. The re-evaluation showed that of the twelve sample sets that were positive for BV according to the modified Hay/Ison criteria during the first evaluation but negative for the molecular test seven were still regarded as BV positive and five were re-classified as non-BV. Out of the eight sample sets positive for BV on the molecular tests but negative using the modified Hay/Ison criteria, one wet smear was reclassified as BV positive. After these corrections, a new kappa coefficient value of 0.87 was calculated with a confidence level of 0.76-0.99. For the molecular test, the re-calculated sensitivity was 0.91 and specificity 0.97 . The re-calculated positive predictive value was 0.91 and the negative predictive value was 0.97 (Table 2). One interesting observation made during the re-evaluation of the slides was that of the twelve that originally tested positive for BV on wet smear but negative in PCR, seven contained sperm.

\begin{tabular}{|l|l|l|l|l|}
\hline & \multicolumn{3}{|c|}{ Molecular test } & \\
\hline & & BV & Normal & \\
\hline \multirow{2}{*}{$\begin{array}{l}\text { Modified Hay/ } \\
\text { Ison }\end{array}$} & BV & 69 & 7 & 76 \\
\cline { 2 - 6 } & Normal & 7 & 205 & 212 \\
\hline & & 76 & 212 & 288 \\
\hline
\end{tabular}

Table 2. After re-evaluation of the air-dried wet smears the kappa coefficient value was recalculated $\kappa=0.87(0.76-0.99)$ between the modified Hay/Ison criteria for the diagnosis of BV and a molecular test using 6 different pathogens and an algorithm using the presence of lactobacilli. This gives a sensitivity of $0.91(0.82-$ $0.95)$, a specificity of $0.97(0.93-0.98)$, positive predictive value of $0.91(0.82-0.95)$ and negative predictive value of 0.97 (0.93-0.98). 


\section{Discussion}

Vaginal discomfort is a common issue experienced during a woman's menses. BV is an aggravating condition that doesn't necessarily require treatment to clear an infection in non-pregnant healthy women, treatment serves only to relieve discomfort. In pregnant women or women undergoing gynecological procedures, however, treatment for BV is likely to decrease the risk of premature delivery [19] or postoperative complications [20].

When examining the cause of vaginal discomfort the current recommendation is to use microscopy in conjunction with the Hay/Ison criteria for clinical diagnosis of BV [21].This method for diagnosis of $\mathrm{BV}$, as with the Amsel's criteria and the Nugent's score, suffers from reliability on subjective judgments [4], questionable reproducibility, high economic costs and are heavily time-consuming methods requiring highly skilled technicians and physician consultation for sampling and, more importantly, these methods are seldom used to their full extent, except in research. There is therefore a great need for an easy and objective method to assess BV in out-patient care.

The results of our study indicate that it is safe to replace a clinical evaluation of an air-dried wet vaginal smear using the modified Hay/Ison criteria with the investigated molecular PCR test for the diagnosis of BV. The kappa coefficient value, sensitivity and specificity obtained in this study compares well with other molecular methods [9-13). Another advantage of the molecular test is that sampling can be performed by the women themselves [12]. Thus, if the test is sent to the laboratory by the woman the result can be available at the time of the clinical appointment, which may shorten the time before treatment can commence. Replacing the diagnostic method may also have positive implications for the cost of handling this type of patient. The cost for each test must be compared to the benefits of a decreased demand for physician's examination and time, reduction in the number of late miscarriages and fewer complications associated with abortion. In general, however, performing a test of cure is also important to exclude other reasons for malodorous discharge other than BV such as trichomoniasis, cervical cancer, and candida infection.

In this study all women included were pregnant, which means they were not affected by the normal hormone level changes experienced during the reproductive cycle. It could be of great interest to study a non-pregnant female population and to evaluate if the reliability is as excellent as seen in this study. Furthermore, the molecular test may be a useful tool during treatment follow-up. Treatment of BV can be challenging with high risk for relapse after treatment [22].

One parameter to look further into is whether recent intercourse, identified as presence of sperm in the sample, can affect the result of the molecular test. In this study half (7/12) of the false negative molecular tests contained sperms in the wet smear.

\section{Conclusion}

The studied molecular test that quantifies the presence of six different pathogens and lactobacilli and calculates a single result in a algorithm has, compared with traditional BV diagnosis using the modified Hay/Ison criteria, a kappa value of 0.87 ,a fully acceptable result clinical practice. The molecular test could successfully replace the commonly used Amsel criteria as the criteria are too subjective. If the molecular test will be equally as effective when used as a "test of cure" needs to be investigated.

\section{Abbreviations: BV: Bacterial vaginosis}

\section{Declarations}

Ethical approval for the study was obtained from the regional ethics committee (EPN) for Gothenburg Box 40140530 Göteborg Sweden, University of Gothenburg, Box 100, SE-405 30 Gothenburg, SWEDEN. (EPN reference number 658-09 entitled "Molecular biological methods to verify STD-agents") on 21 January 2010. Participants consented to participate through providing verbal informed consent before their inclusion in the study.

Clinical trail registration \# NCT04067557, retrospectively registered.

\section{Availability of data and materials}

The datasets generated and/or analyzed during the current study are not publicly available due individual privacy could be compromised but are available from the corresponding author on reasonable request.

\section{Competing interests}

P.G.L. has received speaking fees from Campus Pharma AB. This study was conducted in cooperation with Dynamic Code $\mathrm{AB}$, a company with a commercial interest in marketing 
a reliable molecular diagnostic test for bacterial vaginosis. They provided all the materials needed for collecting vaginal smears and molecular diagnostic testing. M.F. is employed as a Senior Scientist and A.H. is employed as a Product Developer at Dynamic Code AB. AS is Medical Advisor at Dynamic Code, K.B, I.V and J.L declare that they have no conflicts of interest.

\section{Funding}

This study was partly funded by the Skaraborgs Hospital FoU fund.

\section{Authors' contributions}

All authors have been involved in the writing of the article. PGL is the main author, $\mathrm{KB}$ and JL has been designing the study and IV has administered the study and $\mathrm{AH}$ and MF has also done the molecular analysis, AS developed the algorithm of the tet.

\section{References}

1. Forsum U, Hallen A, Larsson PG (2005) Bacterial vaginosis--a laboratory and clinical diagnostics enigma. APMIS 113: 153-161.

2. Amsel R, Totten PA, Spiegel CA, Chen K, Eschenbach DA, Holmes KK (1983) Nonspecific vaginitis. Diagnostic criteria and microbial and epidemiologic associations. Am J Med 74: 1422.

3. Nugent RP, Krohn MA, Hillier SL (1991) Reliability of diagnosing bacterial vaginosis is improved by a standardized method of gram stain interpretation. J ClinMicrobiol 29: $297-$ 301.

4. Forsum U, Jakobsson T, Larsson PG, Schmidt H, Beverly A, Bjornerem A, et al. (2002) An international study of the interobserver variation between interpretations of vaginal smear criteria of bacterial vaginosis. APMIS 110: 811-818.

5. Forsum U, Larsson PG, Spiegel C (2008) Scoring vaginal fluid smears for diagnosis of bacterial vaginosis: need for quality specifications. APMIS 116: 156-159.

6. Larsson PG, Carlsson B, Fahraeus L, Jakobsson T, Forsum U (2004) Diagnosis of bacterial vaginosis: need for validation of microscopic image area used for scoring bacterial morphotypes. Sex Transm Infect 80: 63-67.

7. Hay PE, Lamont RF, Taylor-Robinson D, Morgan DJ, Ison C, Pearson J (1994) Abnormal bacterial colonisation of the genital tract and subsequent preterm delivery and late miscarriage. BMJ 308: 295-298.

8. Ison CA, Hay PE (2002) Validation of a simplified grading of Gram stained vaginal smears for use in genitourinary medicine clinics. Sex Transm Infect 78: 413-415.

9. Menard JP, Fenollar F, Raoult D, Boubli L, Bretelle F (2012) Self-collected vaginal swabs for the quantitative realtime polymerase chain reaction assay of Atopobiumvaginae and Gardnerella vaginalis and the diagnosis of bacterial vaginosis. Eur J ClinMicrobiol Infect Dis 31: 513-51q8.

10. Cartwright CP, Lembke BD, Ramachandran K, Body BA, Nye MB, Rivers CA, et al. (2012) Development and validation of a semiquantitative, multitarget PCR assay for diagnosis of bacterial vaginosis. J ClinMicrobiol 50: 2321-2329.

11. Shipitsyna E, Zolotoverkhaya E, Chen CY, Chi KH, Grigoryev A, Savicheva A, et al. (2013)Evaluation of polymerase chain reaction assays for the diagnosis of Trichomonas vaginalis infection in Russia. J EurAcad Dermatol Venereol 27: e217-223. 12. Gaydos CA, Beqaj S, Schwebke JR, Lebed J, Smith B, Davis TE, et al. (2017) Clinical Validation of a Test for the Diagnosis of Vaginitis. Obstet Gynecol 130: 181-189. 
13. Schwebke JR, Gaydos CA, Nyirjesy P, Paradis S, Kodsi S, Cooper CK (2018) Diagnostic Performance of a Molecular Test versus Clinician Assessment of Vaginitis. J ClinMicrobiol 56.

14. Carlsson I, Breding K, Larsson PG (2018) Complications related to induced abortion: a combined retrospective and longitudinal follow-up study. BMC Womens Health 18:158.

15. Charonis G, Larsson PG (2006) Use of $\mathrm{pH} /$ whiff test or QuickVue Advanced $\mathrm{pH}$ and Amines test for the diagnosis of bacterial vaginosis and prevention of postabortion pelvic inflammatory disease. Acta ObstetGynecol Scand 85: 837-843.

16. Larsson PG, Platz-Christensen JJ (1990) Enumeration of clue cells in rehydrated air-dried vaginal wet smears for the diagnosis of bacterial vaginosis. Obstet Gynecol 76: 727-730.

17. Larsson P-G, Breding K, Vikström I, Larsson J (2018) Validation of Hay/Ison Criteria for A Diagnosis of Bacterial Vaginosis Using an Air-Dried Wet Smear. J JGynecObst 5: 038.

18. Banerjee M, Capozzoli M, McSweeney L, Sinha D (1999) Beyond kappa: A review of interrater agreement measures. CMJ 27: 3-23.

19. Lamont RF, Nhan-Chang CL, Sobel JD, Workowski K, Conde-Agudelo A, Romero R (2011) Treatment of abnormal vaginal flora in early pregnancy with clindamycin for the prevention of spontaneous preterm birth: a systematic review and metaanalysis. Am J Obstet Gynecol 205: 177-190.

20. Larsson PG, Bergström M, Forsum U, Jacobsson B, Strand A, Wolner-Hanssen P (2005) Bacterial vaginosis. Transmission, role in genital tract infection and pregnancy outcome: an enigma. APMIS 113: 233-45.

21. Sherrard J, Wilson J, Donders G, Mendling W, Jensen JS (2018) European (IUSTI/WHO) International Union against sexually transmitted infections (IUSTI) World Health Organisation (WHO) guideline on the management of vaginal discharge. Int J STD AIDS13: 1258-1272.

22. Larsson PG, Brandsborg E, Forsum U, Pendharkar S, Andersen KK, Nasic S, et al. (2011) Extended antimicrobial treatment of bacterial vaginosis combined with human lactobacilli to find the best treatment and minimize the risk of relapses. BMC Infect Dis 11: 223.
Submit your manuscript to a JScholar journal and benefit from:

- Convenient online submission

ฯ Rigorous peer review

- Immediate publication on acceptance

q Open access: articles freely available online

9. High visibility within the field

- Better discount for your subsequent articles

Submit your manuscript at http://www.jscholaronline.org/submit-manuscript.php 